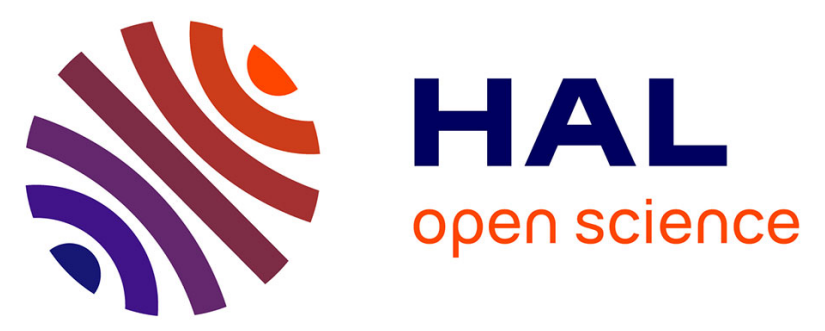

\title{
Comportement de la reconstruction latérale du genou lors d'une flexion de genou en charge et d'un pivot-shift : une étude de simulation
}

Yoann Blache, Biova Kouevidjin, Jacques de Guise, Raphaël Dumas, Adnan Saithna, Bertrand Sonnery-Cottet, Mathieu Thaunat

\section{To cite this version:}

Yoann Blache, Biova Kouevidjin, Jacques de Guise, Raphaël Dumas, Adnan Saithna, et al.. Comportement de la reconstruction latérale du genou lors d'une flexion de genou en charge et d'un pivot-shift: une étude de simulation. Revue de Chirurgie Orthopédique et Traumatologique, 2019, 105, pp.446 451. 10.1016/j.rcot.2019.04.005 . hal-03486533

\section{HAL Id: hal-03486533 https://hal.science/hal-03486533}

Submitted on 20 Dec 2021

HAL is a multi-disciplinary open access archive for the deposit and dissemination of scientific research documents, whether they are published or not. The documents may come from teaching and research institutions in France or abroad, or from public or private research centers.
L'archive ouverte pluridisciplinaire HAL, est destinée au dépôt et à la diffusion de documents scientifiques de niveau recherche, publiés ou non, émanant des établissements d'enseignement et de recherche français ou étrangers, des laboratoires publics ou privés.

\section{(ㅇ)(1) $\$$}

Distributed under a Creative Commons Attribution - NonCommerciall 4.0 International 


\section{Mémoire Original}

Comportement de la reconstruction latérale du genou lors d'une flexion de genou en charge et d'un pivot-shift : une étude de simulation

\section{Lateral Extra-Articular Reconstruction Length Changes During Weightbearing Knee Flexion and Pivot Shift: A Simulation Study}

Yoann Blache ${ }^{1}$, Biova Kouevidjin ${ }^{2}$, Jacques de Guise ${ }^{3}$, Raphaël Dumas ${ }^{4}$, Adnan Saithna ${ }^{5,6}$, Bertrand Sonnery-Cottet ${ }^{2}$, Mathieu Thaunat ${ }^{2}$

${ }^{1}$ Univ Lyon, Université Claude Bernard Lyon 1; Laboratoire Interuniversitaire de Biologie de la Motricité - EA 7424, France

${ }^{2}$ Centre Orthopédique Santy- Group Ramsay-Générale de Santé - Hôpital privé Jean Mermoz, Lyon, France

${ }^{3}$ Laboratoire de recherche en imagerie et orthopédie (LIO), Centre de recherche du Centre hospitalier de l'Université de Montréal (CRCHUM), Montréal , Canada

${ }^{4}$ Univ Lyon, Université Claude Bernard Lyon 1, IFSTTAR, LBMC UMR_T9406, F69622 Lyon, France

${ }^{5}$ Southport \& Ormskirk Hospitals, Lancashire, UK

${ }^{6}$ Department of Clinical Engineering, University of Liverpool, Merseyside, UK

\section{$\underline{\text { Auteur correspondant }}$}

Blache Yoann

Univ Lyon, Université Claude Bernard Lyon 1

Laboratoire Interuniversitaire de Biologie de la Motricité - EA 7424

27-29 bd du 11 novembre 1918, 69622 Villeurbanne Cedex, France

yoann.blache@univ-lyon1.fr

Ne pas utiliser, pour citation, la référence française de cet article, mais celle de l'article original paru dans Orthopaedics \& Traumatology: Surgery \& Research, en utilisant le DOI ci-dessus. 


\section{Résumé}

Introduction : Le comportement d'une reconstruction latérale extra-articulaire (LER) a été largement étudié lors de la flexion du genou, alors qu'aucune information n'est donnée lors d'un pivot-shift. Ainsi l'objectif de cette étude était d'évaluer les variations de tension d'une LER lors d'une tache de flexion de genou en charge et lors de scenarios de pivot-shift simulés.

Hypothèse : un tunnel fémoral postérieur et proximal à l'épicondyle fémoral latéral permettait à la LER de se tendre dans les premiers degrés de flexion de genou, lors de la flexion en charge et lors de pivot-shift simulés.

Matériel et méthode : Un modèle informatique a été implémenté pour simuler des flexions en charge et des scenarios de pivot-shift. La mise en tension de la LER a été calculé dans les deux conditions en estimant la distance entre six localisations de tunnels fémoraux (tous postérieurs et proximal à l'épicondyle fémoral) et deux postions de tunnels tibiaux : le tubercule de Gerdy (GT) et l'insertion anatomique du ligament antérolatéral natif (ALL).

\section{Résultats}

Indépendamment de la position des tunnels fémoraux ou tibiaux, la LER se tendait jusqu'à $22 \%$ de la valeur de repos lors des premiers degrés de flexion de genou en charge, puis se relâchait de 40 à $60^{\circ}$ de flexion de genou. Cependant, un tunnel tibial localisé à l'insertion du ligament antéro latéral (ALL) permettait un relâchement complet de la LER à $60^{\circ}$ de flexion de genou alors que lorsque le tunnel était placé en GT la LER restait plus tendue. Pour les mouvements de pivot-shift simulés et quelle que soit la position du tunnel fémoral, la LER se tendait entre 20 et $34 \%$ de la valeur de repos pour un tunnel tibial en GT et entre 11 et $26 \%$ pour un tunnel tibial en ALL. 


\section{Discussion}

Lors d'une flexion de genou en charge, un tunnel fémoral postérieur et proximal à l'épicondyle fémoral permettait à la LER de se tendre lors des premiers degrés de flexion et de se relâcher entre 40 et $60^{\circ}$ de flexion. Lors de pivot-shifts simulés la LER se tendait, supportant ainsi la notion qu'un positionnement postéro-proximal du tunnel fémoral de la LER serait efficace à la fois lors d'une flexion de genou en charge et lors d'une cinématique altérée de genou.

Mots clés : Genou ; ligament antérolatéral ; ligament croisé antérieur ; modèle informatique ; biomécanique 


\section{Introduction}

Le retour au sport après une reconstruction du ligament croisé antérieur n'est pas toujours satisfaisant puisque seulement $63 \%$ des patients retrouvent le niveau de pratique qu'ils avaient avant la lésion [1] et ces derniers sont plus sujets à une nouvelle rupture [2-4]. Une performance altérée associée à un risque accru de re-rupture s'expliquerait par un manque persistant du contrôle de la stabilité du genou même après reconstruction $[5,6]$. Dans ce contexte, la reconstruction latérale extra-articulaire (LER) génère de plus en plus d'intérêt et est de plus en plus réalisée [7-11] afin d'améliorer le contrôle de la rotation et de la translation antérieure du tibia par rapport au fémur [12-14].

La littérature met en exergue un débat important concernant l'anatomie et la fonctionnalité des structures antérolatérales du genou. Néanmoins, un consensus émerge, montrant que le ligament antérolatéral natif s'insérerait en proximal et distal de l'épicondyle fémoral, et entre la tête de la fibula et du tubercule de Gerdy pour le tibia. Le ligament antérolatéral natif présenterait un comportement anisométrique, se tendant en extension et se relâchant en flexion de genou [12,15,16].

Le débat persiste concernant la position optimale des tunnels fémoraux et tibiaux pour fixer le ligament reconstruit. La LER devrait être placée de manière à conférer une cinématique du genou physiologique, surtout en restaurant le contrôle de la rotation mais en évitant de sur contraindre le genou [12]. La littérature incluant des études in-vitro et in-vivo rapporte un large éventail de positions des tunnels fémoraux allant de positions proximales $[10,17,18]$, postero-proximales [13,19] ou encore postero-distales [20]. Cependant, un consensus semble naitre, stipulant qu'une position postero-proximale à l'épicondyle fémoral permettrait un comportement de la LER le plus vertueux possible, à savoir, une LER qui se tend en extension et qui se relâche lors de la flexion du genou [12,20]. Concernant le tunnel 
tibial, une précédente étude [18] a montré que lors d'une flexion de genou en charge, le point le plus isométrique se situerait à $37 \%$ de la largeur du plateau tibial.

Bien que toutes ces études donnent des informations précieuses pour les chirurgiens au regard de la position optimale de la LER en flexion d'un genou physiologique, aucune d'elles n'a évalué les variations de longueur de la LER lorsque la cinématique du genou est altérée avec notamment une laxité en rotation interne et translation antérieure persistante après une reconstruction du ligament croisé antérieur. A notre connaissance, seul Imbert et al [13] ont évalué l'effet d'une rotation du genou sur la variation de longueur de la LER. Parmi, les trois positions fémorales testées, une position postero-proximale présentait la plus grande variation de longueur de la LER lorsque le tibia était soumis à un moment de force en rotation interne de 2 N.m. Cependant cette étude réalisée in-vitro ne reflète peut-être pas le comportement de la LER lors une flexion de genou en charge. De plus, les variations de longueur de la LER n'ont jamais été mesurées lors d'une combinaison de rotation interne et de translation antérieure du tibia qui correspondrait à une instabilité observée lors d'un test de pivot-shift. Un comportement vertueux attendu de la LER serait alors que cette dernière se tende lors d'un test de pivot-shift afin de contrôler l'instabilité en rotation et translation.

L'objectif de cette étude était d'évaluer les variations de tension de la LER lors d'une flexion de genou physiologique en charge et lors d'une simulation de plusieurs scenarios de pivot-shift. Les hypothèses étaient qu'une position fémorale postéro-proximale permettrait à la LER de se tendre proche de l'extension, de se relâcher en flexion et de se tendre lors d'une cinématique altérée comme rencontrée lors d'un scenario de pivot-shift.

\section{Patients and Methods}

\subsection{Modèle informatique}


Un modèle informatique du genou adapté d'Opensim a été implémenté (Opensim 3.3, Delp et al [21]). La géométrie osseuse du modèle du genou provenait d'un CT-Scan (tranches de $6 \mathrm{~mm}$, Siemens Somaton, Erlangen, Germany) d'un homme sain volontaire (Age : 35 ans, Taille : $1.75 \mathrm{~m}$, Masse : $80 \mathrm{Kg}$ ). Le participant n'avait déclaré aucun antécédent de traumatisme des membres inférieurs et avait signé un consentement écrit. Le protocole avait été au préalable approuvé par le comité d'éthique de «l'Hopital Privé Jean Mermoz» (\# 2017-02). Le modèle informatique était également composé d'un objet virtuel sphérique afin d'assurer que les ligaments contournaient l'épicondyle latéral fémoral et ne traversaient pas les os.

De plus, des images biplanaires EOS® (EOS Imaging Inc., Paris, France) du genou du participant ont été utilisées pour évaluer la cinématique du genou lors de la flexions en charge durant cinq positions quasi-statiques : $0,10,20,30$ et $60^{\circ}$ de flexion de genou (un enregistrement par position) [22]. Par la suite une interpolation a été réalisée (Matlab, MathWorks Inc., Natick, MA) afin d'introduire dans le modèle les couplages articulaires entre la flexion du genou et les cinq autres degrés de liberté (rotation interne/externe, abduction/adduction, translation cranio-caudal, translation antéro-postérieure, translation médio-latérale) [23].

\subsection{Positions des tunnels simulés}

En suivant le consensus du groupe d'experts sur le ligament antérolatéral [12], les tunnels fémoraux étaient localisés en postérieur et proximal au condyle fémoral latéral. Plus précisément, six tunnels fémoraux ont été simulés, placés sur deux quarts de cercle ayant pour rayon 5 et $10 \mathrm{~mm}$, et étant centrés au niveau de l'épicondyle fémoral latéral (Figure 1). Pour le tibia, deux localisations ont été considérées. Le premier tunnel répliquait la procédure utilisant la bandelette ilio-tibiale, en étant situé sur le tubercule de Gerdy (GT). Le second 
tunnel tibial répliquait la position antérieure du ligament antérolatéral (ALL) soit à $1 \mathrm{~cm}$ en arrière du tubercule de Gerdy [7] (Figure 1).

\subsection{Analyse}

La longueur de la LER a été calculée en supposant que le ligament restait en contact permanent avec les structures rigides. Dans cette configuration, un raccourcissement de la LER traduisait un relâchement de la LER, alors qu'un allongement de la LER correspondait à un ligament tendu. La variation de longueur de LER estimée lors des flexions en charge a été normalisée par rapport à la longueur de la LER lorsque le genou était en extension complète (Eq. 1)

$$
\Delta L E R_{\text {longueur }}=\frac{\left(L E R_{\text {longueur }}^{i, j, k}-L E R_{\text {longueur_conplète }}^{\text {Extension_con }}\right)}{L E R_{\text {longueur }}^{\text {Extensiomplète }}}
$$

avec, i, j, k correspondant à une position de genou composée respectivement d'une flexion, rotation interne et translation antérieure.

Dans un second temps, la longueur de LER a été calculée lors de cinématiques de genou altérées, à savoir une exagération de la rotation interne (jusqu'à $8^{\circ}$ ) et de la translation antérieure (jusqu'à $12 \mathrm{~mm}$ ) du tibia [24]. Enfin, la variation de longueur moyenne de la LER a été calculée pour deux volumes représentatifs d'une cinématique du genou rencontrée lors d'un test de pivot-shift. Le premier volume correspondait à une combinaison de flexion de genou (entre 10 et $30^{\circ}$ ), de rotation interne (entre 1 et $8^{\circ}$ ) et de translation antérieure (entre 1 et $6 \mathrm{~mm}$ ). Le second volume testé correspondait à la même plage angulaire en flexion et en rotation mais avec une translation allant de 7 à $12 \mathrm{~mm}$. Enfin une combinaison spécifique de 
flexion, rotation interne et translation antérieure du genou a été simulée pour reproduire les courbes de pivot-shift présentées par Amis et al [5] (Figure 2).

\section{Résultats}

En charge sur genou normal, la flexion du genou s'accompagnait d'une rotation interne comprise entre 0 et $21,7^{\circ}$ et d'une translation antérieure comprise entre 0 et $6,3 \mathrm{~mm}$ (Figure 2). Lors de cette épreuve, la LER se tendait (allongement) jusqu'à $22 \%$ de la longueur de repos dans les premiers degrés de flexion et se relâchait (raccourcissement) entre 40 et $60^{\circ}$ de flexion de genou quelle que soit la position du tunnel fémoral et tibial (Figure 3). Quelle que soit la position, du tunnel fémoral, la LER était moins tendue lorsque le greffon était fixé sur la partie postérieure du tibia (ALL) que sur le GT (- $7.72 \pm 0.77 \%$ en moyenne).

Dans le cas de simulations de cinématiques de genou altérées, une augmentation de la rotation interne du tibia, en particulier après $20^{\circ}$ de flexion de genou, amenait la LER à se tendre indépendamment de la position des tunnels. De façon analogue, la LER se tendait lorsque qu'une translation antérieure du tibia était simulée (Figure 4 et document supplémentaire). Pour les volumes spécifiques d'une cinématique rencontrée lors d'un test de pivot-shift (genou en flexion entre 10 et $30^{\circ}$ associé à une rotation interne et une translation antérieure du tibia) la LER se tendait entre 20 et $34 \%$ de la valeur de repos lorsque le tunnel tibial était antérieur (GT) et entre 11 et $26 \%$ pour un tunnel tibial plus postérieur (ALL) (Tableau 1). Alors que la position du tunnel tibial influençait la tension de la LER, de très faibles différences étaient observées entre les différentes positions du tunnel fémoral tant qu'ils étaient postérieur et proximal à l'épicondyle fémoral (i.e. moins de 1,4 $\mathrm{mm}$ et 2,3 $\mathrm{mm}$ de variation pour GT et ALL respectivement).

Enfin, lorsque la cinématique du genou selon Amis et al [5] lors d'un test de pivot shift était simulée, la LER se tendait au début du test (jusqu'à $15^{\circ}$ de flexion du genou). Les tensions 
maximales étaient observées lors des pics de rotation interne et de translation antérieure du tibia quelle que soit la localisation des tunnels fémoraux.

\section{Discussion}

Cette étude montre tout d'abord qu'un tunnel fémoral postérieur et proximal à l'épicondyle fémoral latéral permet à la LER de se tendre lors des premiers degrés de flexion et de se relâcher entre 0 et $60^{\circ}$ lors de flexion de genou en charge. Le second résultat est que lorsque le tunnel est postérieur et proximal, la LER se tend lors d'un test de pivot-shift simulé. Enfin, lorsque la LER est fixée en GT, cette dernière est plus tendue à la fois lors d'une flexion en charge et lors d'un scénario de pivot-shift en comparaison à une fixation en ALL.

Bien que cette étude se fonde uniquement sur un participant, la cinématique du genou mesurée lors de la flexion de genou en charge est représentative d'une population saine. Une cinématique de genou physiologique lors d'une flexion en charge se caractérise par une rotation interne et une translation antérieure du tibia [25]. Nos résultats sont donc en accord avec la littérature puisque nous avons observé une rotation interne de 0 à $20^{\circ}$ et une translation antérieure de 0 à 6 mm associée à la flexion de genou en charge.

Une LER est réalisée afin d'améliorer le contrôle de la stabilité du genou en rotation et translation $[12,26]$. Comme montré par la littérature, notre étude met en avant que des tunnels fémoraux localisés postérieurement et proximalement à l'épicondyle fémoral latéral favorisent une LER tendue en extension et relâchée en flexion $[6,13,20]$. La LER se tend également à la fois avec une rotation interne et une translation antérieure simulée du tibia De plus, comme nous l'avions déjà montré [20], nous avons observé qu'un tunnel tibial en ALL permettait plus de relâchement de la LER autour de $60^{\circ}$ de flexion que lorsque le greffon était fixé en GT. Ainsi, un tunnel tibial en ALL présenterait un comportement plus vertueux de la LER 
qu'un positionnement en GT puisque cela permettrait de moins contraindre la rotation interne physiologique lors de la flexion du genou.

. Il est intéressant de noter que ce comportement était observé indépendamment de la position du tunnel fémoral tant que celui-ci était postéro-proximal. Ce résultat suggère que les chirurgiens n'ont alors pas la nécessité d'être précis à $5 \mathrm{~mm}$ lorsqu'ils déterminent la positon du tunnel fémoral. Ces résultats sont en accord avec ceux présentés par Imbert et al [13] qui ont montré une mise en tension de la LER lorsque qu'un couple de force était appliqué en rotation interne.

La LER se tendait également lors de tests de pivot-shift simulés, à savoir, une combinaison de rotation interne et de translation antérieure du tibia. Le pic de tension était d'ailleurs observé lorsque la translation antérieure et la rotation interne étaient maximales lorsque le test de pivot-shift présenté par Amis et al [5] était simulé. Ces résultats sont consistants avec ceux présentés par Inderhaug et al [27], qui ont montré une amélioration significative de la cinématique du genou cadavérique après une LER. Ainsi, le comportement de la LER observé dans cette étude tend à confirmer le concept qu'une LER pourrait être indiquée après une rupture du ligament croisé antérieur lorsque le patient présente un pivotshift de grade 2 ou 3 afin d'améliorer le contrôle de la stabilité du genou [12]. Cependant, la mise en tension de la LER est seulement un des facteurs permettant le contrôle complexe de la stabilité du genou. La direction du vecteur de force créé par la LER doit également être étudiée dans de futures études afin de valider que la mise en tension de la LER favorise le contrôle de la stabilité du genou.

Enfin, la comparaison du positionnement tibial en ALL, ou en GT montrait une plus grande tension de la LER lors de simulations de rotations internes et de translations antérieures du tibia. Ce résultat amène à penser qu'un tunnel tibial en GT pourrait surcontraindre le genou en ne favorisant pas un complet relâchement de la LER en flexion. 
Cette étude a plusieurs limites. La première limite est que les conclusions de cette étude se fondent sur un modèle informatique simplifié du genou. Néanmoins, la longueur in-vivo de la LER ne peut être mesurée que pour des positions statiques, puisque qu'aucun outil ne permet actuellement de mesurer le comportement des tissus mous en dynamique. La seconde limite est qu'un seul participant a été impliqué dans cette étude. Cependant, l'analyse effectuée dans cette étude explore une large amplitude de rotations internes et de translations antérieures du tibia qui devrait englober la variabilité interindividuelle qui aurait été observée dans une cohorte de patients. De façon similaire, la multitude de positions fémorales testées compense la variabilité d'anatomie du genou interindividuelle. La troisième limite était que la cinématique altérée du genou a été simulée à partir de données de la littérature, cinématique qui pourrait différer de celle observée chez des patients en post-chirurgie. Mais la grande variabilité de cinématiques testées devrait minimiser cette limite. Enfin seulement deux points d'insertion tibiaux ont été étudiés. Cependant ces deux points tibiaux représentent les tunnels tibiaux les plus utilisés en clinique [7,8].

\section{Conclusion}

La modélisation informatique permet d'analyser la mise en tension de la LER pour de nombreuses positions de genou correspondant à une flexion en charge, et pour la première fois, à une cinématique observée lors d'un pivot-shift. Lors de la flexion de genou en charge, un positionnement postéro-proximal du tunnel fémoral permet à la LER de se tendre lors des premiers degrés de flexion et de se relâcher entre 40 et $60^{\circ}$. Mais un positionnement tibial en GT, engendre un plus grand risque de sur-contrainte du genou. Enfin, lors de pivots-shifts simulés la LER se tend quel que soit le scénario testé. Ces résultats confirment que les chirurgiens peuvent réaliser une LER pour des patients ayant une rupture du ligament croisé antérieur. Lorsqu'une telle chirurgie est réalisée, un positionnement postéro-proximal du 
tunnel fémoral vis-à-vis de l'épicondyle fémoral latéral est conseillé, puisqu'il permet un comportement vertueux de la LER à la fois en flexion en charge ou bien lors de mouvements de genoux altérés.

\section{Remerciements}

Nous souhaitons remercier Thierry Cresson du «Laboratoire de recherche en imagerie et orthopédie, École de technologie supérieure, Centre de recherche du Centre hospitalier de l'Université de Montréal », pour sa contribution concernant la reconstruction des maillages osseux à partir de la radiographie biplanaire. De plus, ce travail a été réalisé avec le support du LABEX PRIMES (ANR-11-LABX-0063) de l'Université de Lyon et avec le programme "Investissements d'Avenir" (ANR-11-IDEX-0007).

\section{Conflit d'intérêt}

Y.B, R.D., B.K. et J.deG. déclarent n'avoir aucun conflit d'intérêt. M.T., B.S. and A.S. sont consultants pour Arthrex.

\section{Financement}

L'étude a été supportée par le groupe "Ramsay Générale de Santé”, Paris, France.

\section{Contribution des auteurs}

Y.B. a conçu la problématique de l'étude ; traité et analysé les données, écrit et approuvé le manuscrit. B.K, R.D. and J.deG ont effectué les expérimentations, traité les données, écrit et approuvé le manuscrit. A.S., B.S. and M.T. ont analysé les données, écrit et approuvé le manuscrit

\section{Références}


1. Ardern $\mathrm{CL}$, Webster $\mathrm{KE}$, Taylor NF, et al. Return to sport following anterior cruciate ligament reconstruction surgery: A systematic review and meta-analysis of the state of play. Br J Sports Med 2011; 45:596-606.

2. Paterno MV, Rauh MJ, Schmitt LC, et al. Incidence of second acl injuries 2 years after primary acl reconstruction and return to sport. Am J Sports Med 2014; 42:1567-73.

3. Salmon L, Russell V, Musgrove T, et al. Incidence and risk factors for graft rupture and contralateral rupture after anterior cruciate ligament reconstruction. Arthroscopy 2005; 21:948-57.

4. Shelbourne KD, Gray T and Haro M. Incidence of subsequent injury to either knee within 5 years after anterior cruciate ligament reconstruction with patellar tendon autograft. Am J Sports Med 2009; 37:246-51.

5. Amis AA, Bull AMJ and Lie DTT. Biomechanics of rotational instability and anatomic anterior cruciate ligament reconstruction. Oper Tech Orthop 2005; 15:29-35.

6 . Lutz C. Role of anterolateral reconstruction in patients undergoing anterior cruciate ligament reconstruction. Orthop Traumatol Surg Res 2018; 104:S47-S53.

7. Sonnery-Cottet $B$, Daggett $M$, Helito $C P$, et al. Combined anterior cruciate ligament and anterolateral ligament reconstruction. Arthrosc Tech 2016; 5:e1253-e9.

8. Slette EL, Mikula JD, Schon JM, et al. Biomechanical results of lateral extra-articular tenodesis procedures of the knee: A systematic review. Arthroscopy 2016; 32:2592-611.

9. Marcacci M, Zaffagnini S, Marcheggiani Muccioli GM, et al. Arthroscopic intra- and extra-articular anterior cruciate ligament reconstruction with gracilis and semitendinosus tendons: A review. Curr Rev Musculoskelet Med 2011; 4:73-7.

10. Krackow KA and Brooks RL. Optimization of knee ligament position for lateral extraarticular reconstruction. Am J Sports Med 1983; 11:293-302.

11. Panisset JC, Pailhe R, Schlatterer B, et al. Short-term complications in intra- and extra-articular anterior cruciate ligament reconstruction. Comparison with the literature on isolated intra-articular reconstruction. A multicenter study by the french arthroscopy society. Orthop Traumatol Surg Res 2017; 103:S231-S6.

12. Sonnery-Cottet B, Daggett M, Fayard JM, et al. Anterolateral ligament expert group consensus paper on the management of internal rotation and instability of the anterior cruciate ligament deficient knee. J Orthop Traumatol 2017; 18:91-106.

13. Imbert $P$, Lutz $C$, Daggett $M$, et al. Isometric characteristics of the anterolateral ligament of the knee: A cadaveric navigation study. Arthroscopy 2016; 32:2017-24.

14. Louis ML, D'Ingrado $P$, Ehkirch FP, et al. Combined intra- and extra-articular grafting for revision acl reconstruction: A multicentre study by the french arthroscopy society (sfa). Orthop Traumatol Surg Res 2017; 103:S223-S9.

15. Getgood A, Brown C, Lording T, et al. The anterolateral complex of the knee: Results from the international alc consensus group meeting. Knee Surg Sports Traumatol Arthrosc 2018;

16. Feagin J, Hirschmann MT and Muller W. Understand, respect and restore anatomy as close as possible! Knee Surg Sports Traumatol Arthrosc 2015; 23:2771-2.

17. Kittl C, Halewood C, Stephen JM, et al. Length change patterns in the lateral extra-articular structures of the knee and related reconstructions. Am J Sports Med 2015; 43:354-62.

18. Wieser K, Furnstahl P, Carrillo F, et al. Assessment of the isometry of the anterolateral ligament in a 3-dimensional weight-bearing computed tomography simulation. Arthroscopy 2017; 33:1016-23.

19. Van de Velde SK, Kernkamp WA, Hosseini A, et al. In vivo length changes of the anterolateral ligament and related extra-articular reconstructions. Am J Sports Med 2016; 44:2557-62.

20. Kernkamp WA, Van de Velde SK, Hosseini A, et al. In vivo anterolateral ligament length change in the healthy knee during functional activities-a combined magnetic resonance and dual fluoroscopic imaging analysis. Arthroscopy 2017; 33:133-9.

21. Delp SL, Anderson FC, Arnold AS, et al. Opensim: Open-source software to create and analyze dynamic simulations of movement. IEEE Trans Biomed Eng 2007; 54:1940-50. 
22. Clement J, Dumas R, Hagemeister N, et al. Soft tissue artifact compensation in knee kinematics by multi-body optimization: Performance of subject-specific knee joint models. J Biomech 2015; 48:3796-802.

23. Wu G, Siegler S, Allard P, et al. Isb recommendation on definitions of joint coordinate system of various joints for the reporting of human joint motion--part i: Ankle, hip, and spine. International society of biomechanics. J Biomech 2002; 35:543-8.

24. Bull AM, Earnshaw PH, Smith A, et al. Intraoperative measurement of knee kinematics in reconstruction of the anterior cruciate ligament. J Bone Joint Surg Br 2002; 84:1075-81.

25. Yamaguchi S, Gamada K, Sasho T, et al. In vivo kinematics of anterior cruciate ligament deficient knees during pivot and squat activities. Clin Biomech 2009; 24:71-6.

26. Imbert $P$, Lustig $S$, Steltzlen $C$, et al. Midterm results of combined intra- and extra-articular acl reconstruction compared to historical acl reconstruction data. Multicenter study of the french arthroscopy society. Orthop Traumatol Surg Res 2017; 103:S215-S21.

27. Inderhaug E, Stephen JM, Williams A, et al. Anterolateral tenodesis or anterolateral ligament complex reconstruction: Effect of flexion angle at graft fixation when combined with acl reconstruction. Am J Sports Med 2017; 363546517724422. 
Table 1. Moyenne \pm déviation standard de la variation de longueur de la LER (en valeur absolue et relative) lors de deux volumes spécifiques correspondant à des cinématiques de pivot-shift. Les résultats sont reportés pour six simulations de tunnels fémoraux (\#1 à \#6) et deux tunnels tibiaux (GT et ALL).

\begin{tabular}{|c|c|c|c|c|c|c|}
\hline \multirow[b]{2}{*}{$\begin{array}{l}\text { Cinématique altérée } \\
\left(+1 \text { to } 8^{\circ} \text { en rotation) }\right.\end{array}$} & \multicolumn{6}{|c|}{ Positions des tunnels fémoraux } \\
\hline & $\# 1$ & $\# 2$ & \#3 & $\# 4$ & $\# 5$ & $\# 6$ \\
\hline \multicolumn{7}{|l|}{+1 to $6 \mathrm{~mm}$ en translation } \\
\hline $\mathrm{LER}_{\mathrm{GT}} \Delta$ longueur $(\mathrm{mm})$ & $10.8 \pm 1.2$ & $11.1 \pm 1.3$ & $11.3 \pm 1.3$ & $10.6 \pm 1.2$ & $11.2 \pm 1.3$ & $11.3 \pm 1.4$ \\
\hline $\mathrm{LER}_{\mathrm{ALL}} \Delta$ longueur $(\mathrm{mm})$ & $6.0 \pm 1.3$ & $6.6 \pm 1.1$ & $6.9 \pm 1.1$ & $5.9 \pm 0.9$ & $7.0 \pm 1.1$ & $7.5 \pm 1.2$ \\
\hline $\mathrm{LER}_{\mathrm{GT}} \Delta$ longueur $(\%)$ & $22 \pm 2$ & $23 \pm 3$ & $25 \pm 3$ & $20 \pm 2$ & $21 \pm 2$ & $23 \pm 3$ \\
\hline $\operatorname{LER}_{\mathrm{ALL}} \Delta$ longueur $(\%)$ & $13 \pm 2$ & $14 \pm 2$ & $16 \pm 3$ & $11 \pm 2$ & $14 \pm 2$ & $17 \pm 3$ \\
\hline \multicolumn{7}{|l|}{+7 to $12 \mathrm{~mm}$ en translation } \\
\hline $\mathrm{LER}_{\mathrm{GT}} \Delta$ longueur $(\mathrm{mm})$ & $14.8 \pm 1.3$ & $15.4 \pm 1.4$ & $15.7 \pm 1.4$ & $14.5 \pm 1.2$ & $15.6 \pm 1.4$ & $15.9 \pm 1.5$ \\
\hline $\mathrm{LER}_{\mathrm{ALL}} \Delta$ longueur $(\mathrm{mm})$ & $9.3 \pm 1.1$ & $10.2 \pm 1.2$ & $10.7 \pm 1.3$ & $9.1 \pm 1.1$ & $10.8 \pm 1.3$ & $11.6 \pm 1.3$ \\
\hline $\mathrm{LER}_{\mathrm{GT}} \Delta$ longueur $(\%)$ & $30 \pm 3$ & $32 \pm 3$ & $34 \pm 3$ & $27 \pm 2$ & $29 \pm 3$ & $33 \pm 3$ \\
\hline $\mathrm{LER}_{\mathrm{ALL}} \Delta$ longueur $(\%)$ & $20 \pm 2$ & $22 \pm 3$ & $25 \pm 3$ & $17 \pm 2$ & $22 \pm 2$ & $26 \pm 3$ \\
\hline
\end{tabular}




\section{Légendes des figures}

Figure 1. Vue latérale du genou droit avec les localisations des tunnels fémoraux et tibiaux pour les simulations de reconstruction latérale extra-articulaire. ALL : position anatomique du ligament antérolatéral, GT : tubercule de Gerdy.

Figure 2 - Rotation interne (ligne continue bleue) et translation antérieure (ligne continue rouge) du tibia en fonction de la flexion du genou en charge. Les zones bleues et rouges représentent les positions de genou lorsque la rotation interne et/ou la translation antérieure sont exagérées. Les lignes bleues et rouges discontinues correspondent à la cinématique du genou lors d'un test de pivot-shift décrit par Amis et al [5] avec le sens du mouvement décrit par la flèche noire en pointillés.

Figure 3- Variation de longueur de la LER, exprimée en valeur relative (à gauche) et absolue (à droite), en fonction de la flexion du genou lors de la flexion en charge pour six tunnels fémoraux simulés (\#1 à \#6) et deux tunnels tibiaux (GT et ALL)

Figure 4 - Variation de longueur de la LER (rouge pour un ligament tendu et vert pour un ligament relâché) en fonction de la rotation et de la flexion du genou pour trois conditions de translation antérieure du tibia: $0 \mathrm{~mm}, 6 \mathrm{~mm}$ et $12 \mathrm{~mm}$. \#4, \#5 and \#6 correspondent à la localisation des tunnels fémoraux alors que GT et ALL représentent celle des tunnels tibiaux. 


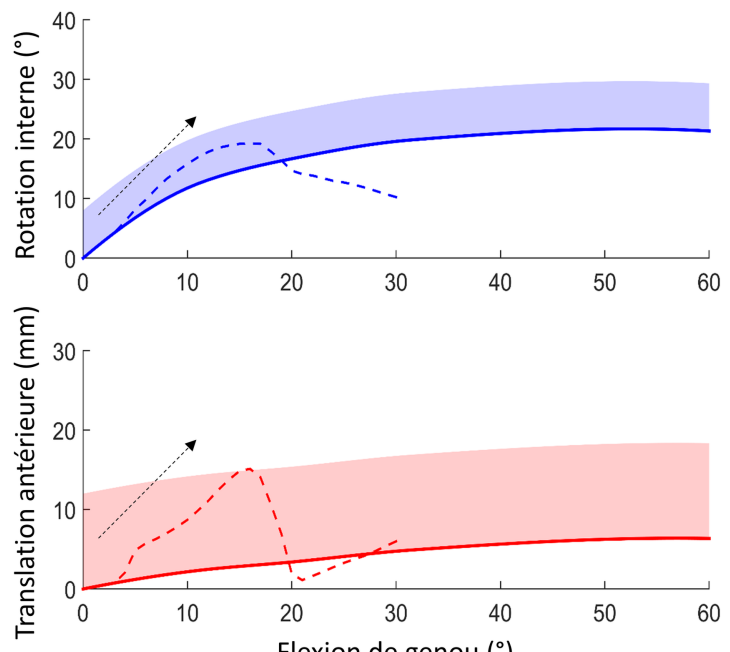

Flexion de genou $\left({ }^{\circ}\right)$ 

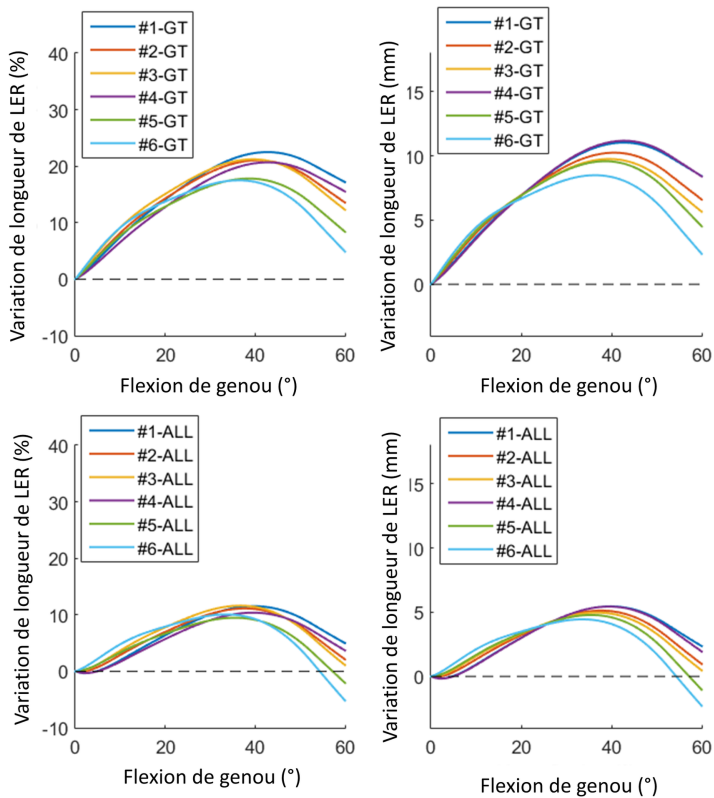
\#4 - GT

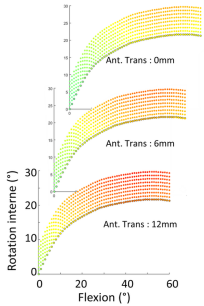

\#4 - ALL

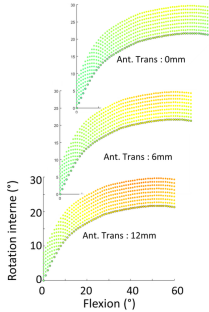

\#5-GT

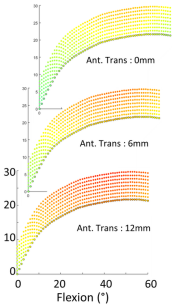

\#5 - ALL

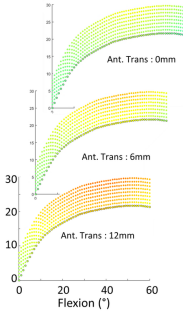

$\Delta$ longueur LER (\%)

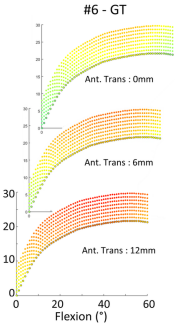

\#6 - ALL

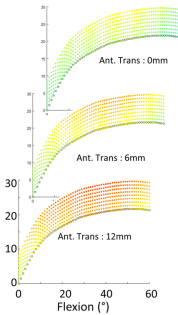

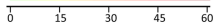

